Trauma Surgery \& Acute Care Open

\title{
Use of prophylactic antibiotic in preventing complications for blunt and penetrating chest trauma requiring chest drain insertion: a systematic review and meta-analysis
}

\author{
Firas Ayoub, Michael Quirke, Daniel Frith
}

Surgery, Queen Mary University of London, London, UK

Correspondence to Dr Firas Ayoub, Surgery, Queen Mary University of London, LondonE1 4NS, UK; fayoub@ rcsi.ie

Received 1 October 2018 Revised 13 January 2019 Accepted 29 January 2019 (c) Author(s) (or their employer(s)) 2019. Re-use permitted under CC BY-NC. No commercial re-use. See rights and permissions. Published by BMJ.

To cite: Ayoub F, Quirke M, Frith D. Trauma Surg Acute Care Open 2019;4:e00246.

\section{ABSTRACT}

Background Chest drain insertion after chest trauma is often associated with high rate of complications. The use of prophylactic antibiotics in patients with blunt and penetrating chest trauma to prevent empyema and pneumonia after chest drain insertion has been debated.

Objective To analyze the effectiveness of prophylactic antibiotics versus placebo to prevent complications in patients with blunt and penetrating chest injuries who require the insertion of a chest drain.

Methods Pubmed, Embase, and grey literature databases were searched during May 2017 for randomized clinical trails comparing prophylactic antibiotic versus placebo in patients with chest injuries requiring chest drain insertion. Good quality randomized studies which met the inclusion criteria were assessed using the Cochrane Collaboration tool for assessing risk of bias and then were included in the systematic review. A meta-analysis of the included studies was concluded using Stata to analyze the relative risk of empyema and pneumonia in these patients.

Results The study identified 12 randomized studies that included 1263 patients with isolated blunt and penetrating chest trauma. The incidence of empyema after a chest drain insertion was 1\% in the antibiotic group and $7.2 \%$ in the placebo group. The incidence of pneumonia after a chest drain insertion was $4.4 \%$ in the antibiotic group and $10.7 \%$ in the placebo group. The use prophylactic antibiotic in those patients was associated with a reduced risk of empyema (relative risk [RR] $0.25 ; 95 \% \mathrm{Cl} 0.13$ to 0.49 ) and pneumonia (RR $0.41 ; 95 \% \mathrm{Cl} 0.24$ to 0.71 ) after chest drain insertion when compared with placebo alone.

Conclusion Prophylactic antibiotic administration in patients with penetrating and blunt chest injuries requiring the insertion of a chest drain was associated with a reduced risk for post-traumatic empyema and pneumonia. Further studies should evaluate the optimal type, dose, and duration of antibiotic given to patients with chest trauma requiring chest drain insertion.

\section{BACKGROUND}

Chest trauma, also known as chest or thoracic injury, is any form of physical injury affecting one or all components of chest wall or thoracic cavity. Chest trauma is common and is responsible for $25 \%$ of all trauma related deaths in the UK. ${ }^{1}$
The vast majority of chest trauma injuries, whether blunt or penetrating, can be managed conservatively in the emergency department using resuscitation and chest drain insertion, and only $10 \%$ to $15 \%$ will require surgical intervention. ${ }^{12}$ Chest drain (chest tube, tube thoracostomy, or intercostal drain) is a fairly simple yet life-saving procedure that involves the insertion of a flexible plastic tube through the chest wall and into the pleural space to allow the drainage of air (pneumothorax) or fluid such as blood (hemothorax). ${ }^{3}$ Chest drain insertion is often associated with a high rate of complication, up to $30 \%$ in some patients. ${ }^{4}$ These are generally divided into insertion, positional, and infective complications.

Post-traumatic empyema and pneumonia are two well-established complications associated with the insertion of a chest drain. Previous studies report that $2 \%-25 \%$ of patients with isolated chest injury who undergo tube thoracostomy could develop infective complications. ${ }^{56}$ The main risk factor for this complication is contamination of the pleural space; mainly at the time of chest drain placement, or from direct penetrating injury to the chest. Other risk factors include perforated viscus with diaphragmatic tear, retained pleural fluid, or spread of remote infection to pleural space. ${ }^{7}$ The use of prophylactic antibiotics in reducing the occurrence of complications associated with insertion of chest drain has been debated. Previous clinical trials have shown mixed outcomes regarding the effectiveness of prophylactic antibiotic in isolated chest trauma. The latest meta-analysis published in 2011 advocates the use of prophylactic antibiotics in penetrating chest trauma. ${ }^{8}$ However, the updated trauma guidelines for antibiotic usage after chest drain insertion published in 2012 by EAST (Eastern Association for the Surgery of Trauma) reported insufficient evidence for prophylactic antibiotic recommendation in patients with chest trauma. ${ }^{9}$

This systematic review is conducted to analyze the effectiveness of prophylactic antibiotics versus placebo to prevent empyema and pneumonia after chest drain insertion in both penetrating and blunt chest injuries. Specifically, we addressed the following points: (1) the incidence of empyema and pneumonia, (2) the length of hospital stay, (3) the duration of chest drain placement. 


\section{METHODS}

This systematic review and meta-analysis was designed, conducted, and reported according to PRISMA (Preferred reporting items for systematic reviews and meta-analysis) guidelines. ${ }^{10}$ The aim of this study is to analyze the effectiveness of prophylactic antibiotics usage versus placebo to prevent complications in patients with blunt and penetrating chest injuries requiring the insertion of a chest drain.

\section{Literature search}

A systematic literature search was conducted during May 2017 using four electronic databases: Medline, Pubmed, Embase, and Cochrane Library. A separate search for additional literature was conducted using Google's search engine. This search strategy was planned and developed by the first author together with an experienced librarian. The search strategy used combination of the following Mesh terms: (chest trauma) OR (chest injury) OR (thoracic injury) AND (chest drain) OR (tube thoracostomy) OR (inter-costal drain) OR (pleural drain) AND antibiotic. No restriction was applied to language or date of publication. All abstracts that met the search criteria were read and examined. To limit potentially missed publications in the electronic search, all lists of references found in selected abstracts were also searched. For studies published in different languages, we used an online translator ('Google' translator) to translate relevant publications. All studies identified were downloaded to a reference manager (Endnote) for detection of duplicates. The authors have also manually checked the included studies for duplicates.

\section{Study selection}

The inclusion criteria included randomized clinical trials that compared prophylactic antibiotic versus placebo to prevent complications in patients with isolated blunt or penetrating chest trauma requiring chest drain insertion. All other non-randomized clinical trials, studies that did not compare prophylactic antibiotic to placebo, studies that did not report empyema or pneumonia after chest drain insertion, studies without statistical difference between participants, duplicate publications, narrative reviews, or opinions were excluded. No limit was applied to the publication date. Studies published anywhere in the world, both English and non-English publications, were included. Two reviews conducted the eligibility criteria assessment independently by examining titles and abstracts identified by the electronic database search result. Any differences in applying the inclusion or exclusion criteria between reviews were resolved by thorough discussion and mutual agreement.

\section{Data extraction}

Two reviewers performed data extraction of eligible articles independently. The extracted information included study ID, year of publication, country of publication, number of patients, type of injury, type, and dose of prophylactic antibiotic used and duration of antibiotic administration. Primary outcomes included the number of patients who developed post-traumatic empyema and/ or pneumonia after chest drain insertion. Secondary outcomes included length of hospital stay, chest tube (ie, tube thoracostomy) duration, and any other potential relevant outcomes.

Refer to table 1 for characteristics of studies included in the meta-analysis of antibiotic versus placebo after chest drain insertion, table 2 for primary outcome, and table 3 for secondary outcomes.

\section{Quality assessment}

The quality of all included studies was assessed using Cochrane Collaboration tool for assessing risk of bias. ${ }^{11}$ Studies were examined using different parameters that included: randomization process, allocation concealment, blinding, incomplete data, and uniform outcome definition. These parameters were graded as low, high, or unclear. Two reviewers independently graded study quality. Results were then compared and any discrepancy was resolved through relevant discussion. Completed assessments allowed studies to be then classified as high, moderate, or poor quality. The methodological quality and Cochrane risk of bias are outlined in table 4 .

\section{Data analysis}

Selected data outcomes were entered into Microsoft Office Excel spreadsheets for analysis. Statistical analysis was conducted using Stata V.13.0. A simple descriptive analysis of primary outcome was used to describe the incidence of empyema and pneumonia in the prophylactic antibiotic group versus placebo after chest drain insertion. For meta-analysis, a pooled random effect model comparing prophylactic antibiotic versus placebo was used to calculate the relative risk of the occurrence of empyema and pneumonia after chest drain insertion in patients with isolated blunt and penetrating chest injuries. Studies heterogeneity was assessed using $\chi^{2}$ and $\mathrm{I}^{2}$ tests.

Table 1 Characteristics of studies included in the meta-analysis of antibiotic vs placebo after chest drain insertion

\begin{tabular}{|c|c|c|c|c|c|c|}
\hline Reference & Year & Country & Sample size & Injury type & Antibiotic & Duration \\
\hline Grover $^{13}$ & 1977 & USA & 75 & Penetrating & Clindamycin $300 \mathrm{mg}$ six hourly & $>24$ hours \\
\hline Stone ${ }^{6}$ & 1981 & USA & 83 & Penetrating, blunt & Cefamandole $1 \mathrm{~g}$ six hourly & $>24$ hours \\
\hline LeBlanc $^{5}$ & 1985 & USA & 52 & Penetrating, blunt & Cefadyl $1 \mathrm{~g}$ & $>24$ hours \\
\hline Mandal $^{14}$ & 1985 & USA & 80 & Penetrating & Doxycycline $100 \mathrm{mg} 12$ hourly & $>24$ hours \\
\hline LoCurto ${ }^{15}$ & 1986 & USA & 58 & Penetrating, blunt & Cefoxitin $1 \mathrm{~g}$ six hourly & $>24$ hours \\
\hline Brunner $^{20}$ & 1990 & USA & 90 & Penetrating, blunt & Cefazolin six hourly & $>24$ hours \\
\hline Cant $^{16}$ & 1993 & South Africa & 113 & Penetrating & Cefazolin $500 \mathrm{mg}$ eight hourly & 24 hours \\
\hline Nichols ${ }^{19}$ & 1994 & USA & 119 & Penetrating, blunt & Cefonicid $1 \mathrm{~g}$ per day & $>24$ hours \\
\hline Gonzalez $^{17}$ & 1998 & USA & 139 & Penetrating, blunt & Cefazolin $1 \mathrm{~g}$ eight hourly & $>24$ hours \\
\hline Maxwell ${ }^{7}$ & 2004 & USA & 224 & Penetrating, blunt & Cefazolin $1 \mathrm{~g}$ eight hourly & $\geq 24$ hours \\
\hline Villegas Carlos ${ }^{18}$ & 2009 & Mexico & 126 & Penetrating, blunt & Cefazolin $1 \mathrm{~g}$ eight hourly & $>24$ hours \\
\hline Heydari' ${ }^{12}$ & 2014 & Iran & 104 & Blunt & Cefazolin $2 \mathrm{~g}$ & 24 hours \\
\hline
\end{tabular}


Table 2 Primary outcomes

\begin{tabular}{|c|c|c|c|c|c|c|}
\hline \multirow[b]{2}{*}{ Study ID } & \multicolumn{3}{|c|}{ Antibiotic group } & \multicolumn{3}{|c|}{ Placebo group } \\
\hline & Total & Empyema & Pneumonia & Total & Empyema & Pneumonia \\
\hline Grover $^{13}$ & 38 & 1 & 4 & 37 & 6 & 13 \\
\hline Stone $^{6}$ & 40 & 1 & 0 & 43 & 2 & 5 \\
\hline LeBlanc ${ }^{5}$ & 26 & 0 & 1 & 26 & 1 & 1 \\
\hline Mandal $^{14}$ & 40 & 0 & 0 & 40 & 1 & 0 \\
\hline LoCurto ${ }^{15}$ & 30 & 0 & 0 & 28 & 5 & 4 \\
\hline Brunner $^{20}$ & 44 & 0 & 1 & 46 & 6 & 3 \\
\hline Cant $^{16}$ & 57 & 0 & 7 & 56 & 5 & 19 \\
\hline Nichols ${ }^{19}$ & 63 & 0 & 0 & 56 & 4 & 3 \\
\hline Gonzalez ${ }^{17}$ & 71 & 0 & 0 & 68 & 2 & 2 \\
\hline Maxwell ${ }^{7}$ & 153 & 2 & 12 & 71 & 4 & 2 \\
\hline Villegas Carlos ${ }^{18}$ & 63 & 3 & N/A & 63 & 5 & $\mathrm{~N} / \mathrm{A}$ \\
\hline Heydari $^{12}$ & 54 & 0 & 2 & 50 & 1 & 4 \\
\hline Total & 679 & $7(1 \%)$ & $27(4.4 \%)$ & 584 & $42(7.2 \%)$ & $56(10.7 \%)$ \\
\hline
\end{tabular}

Values in parenthesis are percentages.

\section{RESULTS}

Literature search

The electronic search of all databases identified 938 records, of which 62 records were screened for relevance. After reading these 62 abstracts, 43 records were excluded; 25 were not relevant to this systematic review and 18 were reviews only. Nineteen records were examined to identify if they meet the inclusion criteria for statistical analysis. However, 7 out of the 19 did not meet the inclusion criteria (4 non-randomized trials and 3 studies examined the duration of prophylactic antibiotics only). Finally, after completing the exclusion criteria, 12 studies were eligible for the meta-analysis (figure 1).

\section{Characteristics of studies included in meta-analysis}

The included studies in this systematic review were all randomized clinical trails that compared the administration of prophylactic antibiotics versus placebo in patients with isolated chest injuries requiring chest drain insertion. All studies included male and female patients above the age of 12 , except one study by Heydari et al $^{12}$ which included patients above the age of eight.

All studies included patients with either blunt or penetrating chest injuries requiring chest drain insertion. Specifically, nine studies included patients with both penetrating and blunt injuries together. Two studies ${ }^{13} 14$ included patients with penetrating chest injuries only, whereas one study ${ }^{12}$ focused on blunt injury only. Penetrating chest injuries were predominantly due to stabbing and gunshot wounds, whereas bunt chest injuries were due to a mixture of trauma including road traffic accidents. For the purpose of this review, we pooled both blunt and penetrating chest injuries together as a representation of a cohort of chest trauma patients requiring insertion of chest drain.

Table 3 Secondary outcomes

\begin{tabular}{|c|c|c|c|c|c|}
\hline \multirow[b]{2}{*}{ Study ID } & \multicolumn{2}{|c|}{ Hospital stay (days) } & \multicolumn{2}{|c|}{ Duration of chest tube (days) } & \multirow[b]{2}{*}{ Other outcomes } \\
\hline & Abx & Placebo & $A b x$ & Placebo & \\
\hline Grover $^{13}$ & Short & & N/A & & $\begin{array}{l}\text { Abx: less fever, positive pleural fluid and wound } \\
\text { cultures }\end{array}$ \\
\hline Stone ${ }^{6}$ & 8 & 10 & 6 & & $\begin{array}{l}\text { Abx: lower positive pleural fluid and wound } \\
\text { cultures }\end{array}$ \\
\hline LeBlanc $^{5}$ & N/A & & N/A & & Abx: less overall infections \\
\hline Mandal $^{14}$ & 6.83 & 5.5 & 3.75 & 4.33 & \\
\hline LoCurto $^{15}$ & 6.6 & 10.3 & 5.4 & 3.8 & Placebo: 5 thoracotomies for empyema \\
\hline Brunner $^{20}$ & 5.2 & 8.3 & 4.1 & 7.2 & \\
\hline Cant $^{16}$ & 3.9 & 5.6 & 3.5 & 4.1 & $\begin{array}{l}\text { Similar positive pleural fluid cultures } \\
\text { Placebo: } 5 \text { thoracotomies for sepsis }\end{array}$ \\
\hline Nichols $^{19}$ & 6.3 & 7.2 & 4.9 & 5 & $\begin{array}{l}\text { Abx: less fever, WBC count } \\
\text { Placebo: } 1 \text { thoracotomy for empyema }\end{array}$ \\
\hline Gonzalez $^{17}$ & 5.9 & 6.3 & 4.9 & 4.8 & $\begin{array}{l}\text { Abx: more chest tube reinsertion, pleural } \\
\text { effusion }\end{array}$ \\
\hline Maxwell ${ }^{7}$ & 7.9 & & 4.6 & & \\
\hline Villegas Carlos ${ }^{18}$ & N/A & & 6.56 & & \\
\hline Heydari' ${ }^{12}$ & N/A & & 6.8 & & \\
\hline
\end{tabular}

Hospital stay and duration of chest tube are presented in mean number of days.

Abx, antibiotics; WBC, white blood cells. 


\begin{tabular}{llllll}
\hline Table 4 & \multicolumn{5}{l}{ Summary of Cochrane risk of bias } \\
\hline Study ID & Randomization & $\begin{array}{l}\text { Allocation } \\
\text { concealment }\end{array}$ & Blinding & $\begin{array}{l}\text { Incomplete } \\
\text { data }\end{array}$ & $\begin{array}{l}\text { Outcome } \\
\text { definition }\end{array}$ \\
\hline Grover $^{13}$ & Low & Low & Low & Low & Low \\
Stone $^{6}$ & Unclear & Unclear & Low & Low & Low \\
LeBlanc $^{5}$ & Unclear & Unclear & Unclear & Low & Low \\
${\text { Mandal }{ }^{14}}^{15}$ & Low & Low & Unclear & Low & Low \\
LoCurto $^{15}$ & High & Unclear & High & Low & Low \\
Brunner $^{20}$ & High & Unclear & Unclear & Low & Low \\
Cant $^{16}$ & Unclear & Unclear & Low & Low & Low \\
Nichols $^{19}$ & Low & Unclear & Low & Low & Low \\
Gonzalez $^{17}$ & Low & Unclear & Low & Low & Low \\
Maxwell $^{7}$ & Unclear & Low & Low & Low & Low \\
Villegas $^{\text {Carlos }}{ }^{18}$ & Unclear & Unclear & Low & Low & Low \\
Heydari $^{12}$ & Unclear & Unclear & Unclear & Low & Low \\
\hline
\end{tabular}

The type of antibiotic and dose were different in some studies. Specifically, six studies used cefazolin, ${ }^{72}{ }^{15-18}$ a first generation cephalosporin with broad bacterial coverage. The remainder of the included studies used clindamycin, ${ }^{13}$ cefamandole,${ }^{6}$ cefadyl,${ }^{5}$ doxycycline, ${ }^{14}$ cefoxitin, ${ }^{15}$ and cefonicid. ${ }^{19}$ The dose of cefazolin was $2-3$ g per 24 hours. In regards to the total duration of antibiotics, 10 studies involved antibiotic administration that exceeded
24 hours, whereas two studies ${ }^{12} 16$ administered antibiotics for 24 hours only. The overall antibiotic duration, for studies that administered antibiotics for more than 24 hours, lasted until the chest drain was removed. Details of characteristics of studies are summarized in table 1.

\section{Primary outcomes}

This systematic review included a total of 1263 patients with isolated blunt and penetrating chest injuries requiring a chest drain. Total 679 (54\%) patients received prophylactic antibiotics whereas $584(46 \%)$ patients received a placebo. The incidence of post-traumatic empyema after a chest drain insertion was $1 \%$ (7/679) in the prophylactic antibiotic group and 7.2\% (42/584) in the placebo group, with $\mathrm{p}=0.0001$. The incidence of post-traumatic pneumonia after a chest drain insertion was 4.4\% (27/616) in the prophylactic antibiotic group and $10.7 \%(56 / 521)$ in the placebo group, with $\mathrm{p}=0.0001$. Details of primary outcomes are summarized in table 2 .

\section{Secondary outcomes}

We reported all secondary outcomes described in the eligible studies, focusing mainly on length of hospital stay and duration of chest tube placement. Seven studies ${ }^{61315-171920}$ reported a shorter length of hospital stay for patients who received prophylactic antibiotics after the insertion of a chest drain compared with receiving a placebo. One study by Mandal et $a l^{14}$ demonstrated

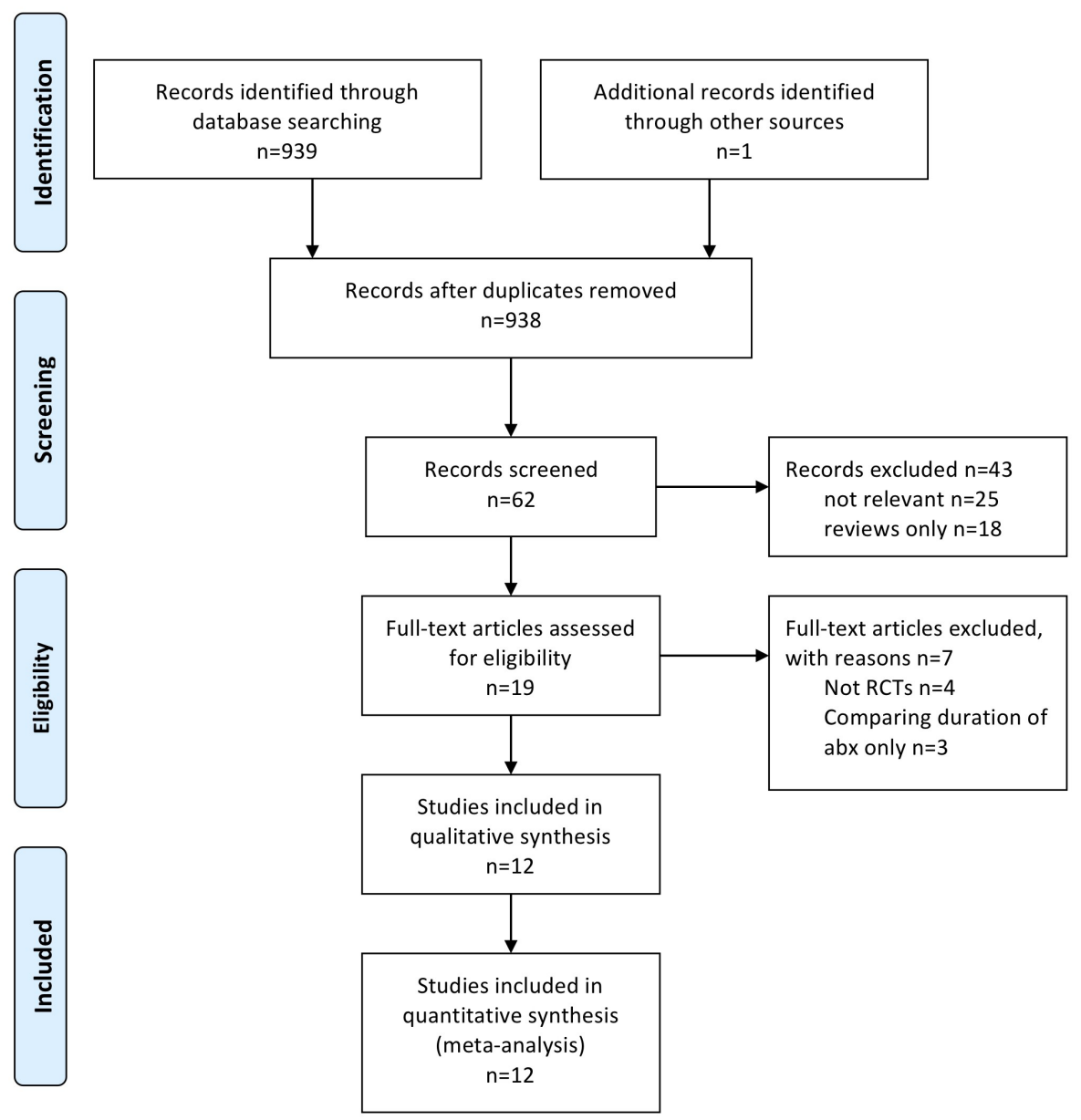

Figure 1 PRISMA flow diagram illustrating the literature search. 
a 6.83day hospital stay in the antibiotic group versus 5.5 days in placebo group. By contrast, Maxwell et al demonstrated a similar length of hospital stay between the two groups.

Nine studies reported a similar duration of chest tube placement between the two groups. However, LoCurto et al $l^{15}$ reported a longer duration of chest tube insertion in the prophylactic group. Interestingly, five studies reported less fever, lower positive pleural fluid and wound cultures, and fewer overall infections in patients who received prophylactic antibiotic versus placebo. More importantly, the number of patients requiring thoracotomy for post-traumatic empyema was significantly higher in the placebo group. ${ }^{15} 1619$ Statistical analysis for secondary outcomes was not possible due to the high heterogeneity in the reported results. Details of secondary outcomes are summarized in table 3 .

\section{Meta-analysis}

On meta-analysis prophylactic antibiotic was associated with overall reduced risk of post-traumatic empyema after chest drain insertion when compared with placebo (relative risk [RR] $0.25 ; 95 \% \mathrm{CI}, 0.13$ to $0.49 ; \chi^{2}$ test $\left.=4.42 ; \mathrm{I}^{2}=0.0 \% ; \mathrm{p}=0.00\right)$ (figure 2A). Similarly, the prophylactic antibiotic group was associated with reduced risk of post-traumatic pneumonia after chest drain insertion when compared with placebo (RR 0.41; 95\% CI 0.24 to $0.71 ; \chi^{2}$ test $=10.12 ; \mathrm{I}^{2}=11.1 \% ; \mathrm{p}=0.002$ ) (figure $2 \mathrm{~B}$ ). This meant that patients with isolated chest injuries who received prophylactic antibiotic after a chest drain insertion had $75 \%$ and $59 \%$ reduced risk of developing post-traumatic empyema and pneumonia, respectively, than patients who received placebo.

\section{DISCUSSION}

Despite increasing evidence supporting the role of prophylactic antibiotics in preventing post-traumatic chest drain complications for patients with chest trauma, there is still a debate concerning its true effectiveness. We performed this study to examine existing randomized clinical trials for evidence concerning the true effectiveness of prophylactic antibiotics against placebo in reducing empyema and pneumonia after chest drain insertion

A

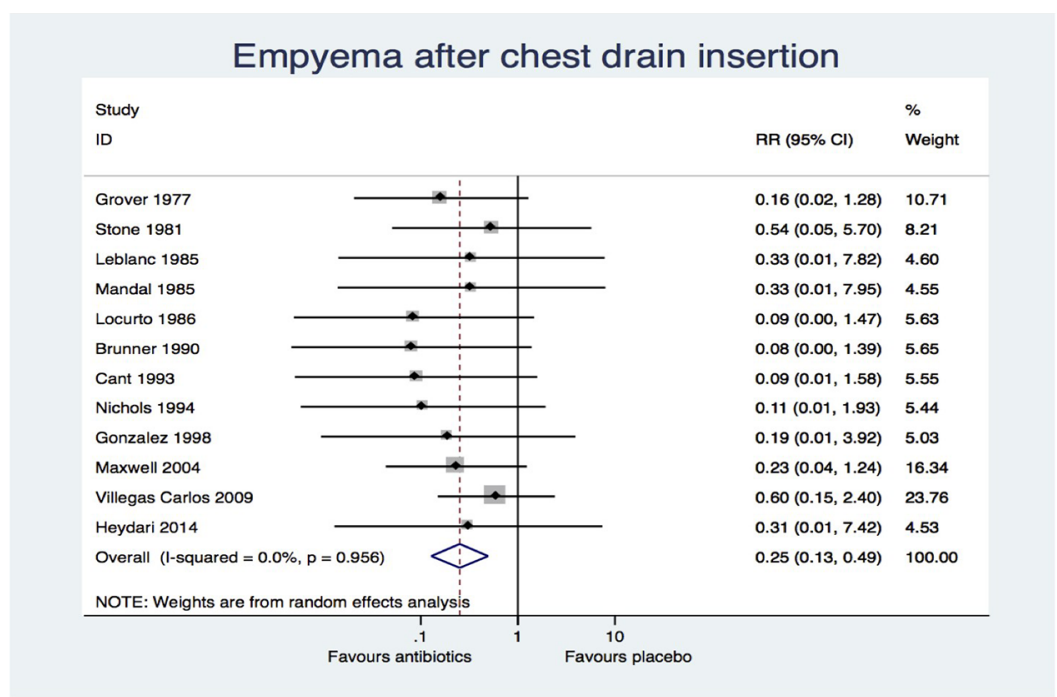

B

\section{Pneumonia after chest drain insertion}

Study

ID

Forest plot showing risk of empyema following chest drain insertion in antibiotic and placebo groups. A random effect model was used for meta-analysis. Relative risk (RR) ratios are shown with $95 \%$ confidence interval $(\mathrm{Cl})$. The vertical line represents the overall pooled estimate.

Figure 2 Meta-analysis comparing antibiotics versus placebo in preventing (a) empyema and (b) pneumonia after chest drain insertion across all studies. 
in patients with blunt and penetrating chest trauma. Using our eligibility criteria and statistical analysis, we found that prophylactic antibiotics reduce the risk of developing empyema and pneumonia in these patients. Meta-analysis of primary outcome demonstrated a lower relative risk for developing empyema and pneumonia in the relevant patient group who received prophylactic antibiotics when compared with placebo alone.

Additionally, length of hospital stay was shown to be shorter in chest trauma patients who were given prophylactic antibiotic when compared with placebo, despite the lack of difference reported in other studies. Even more, the number of thoracotomies required to manage post-traumatic empyema was significantly higher in patients that did not receive prophylactic antibiotics. ${ }^{15} 1619$

The latest meta-analysis, published more than 5 years ago, looked at overall pulmonary infectious complications. ${ }^{8}$ This review concluded that pulmonary complications are less likely to develop in patients with penetrating chest trauma who received prophylactic antibiotic after chest drain insertion. Interestingly, we found only one study, ${ }^{12}$ published in the last 5 years that compared prophylactic antibiotic against placebo in patients with only blunt chest injuries. The latest EAST guidelines published in 2012 did not make recommendation for or against the use of prophylactic antibiotic for chest trauma patients after chest drain insertion. This is because of various factors including previously published studies with mixed qualities, outcomes, and analyses. ${ }^{9}$ However, reviewing recent BTS (British thoracic Society) guidelines $^{21}$ and speaking to trauma experts, prophylactic antibiotic administration after chest drain insertion has been strongly recommended despite the overall low incidence of infectious complication after chest drain insertion.

To our knowledge, this is the first meta-analysis since the EAST guidelines were last updated. We conducted this systematic review according to PRIMSA guidelines ${ }^{10}$ and included randomized clinical trials only, despite some not being double blinded. Our overall risk of bias for most eligible studies is shown to be of high quality.

However, this study is not without limitations. We combined both penetrating and blunt chest injuries in our meta-analysis, despite the pathophysiology and infectious process of penetrating chest injury differing from blunt injury. Penetrating injuries are likely to produce direct injury to the lungs and cause extensive soft tissue disruption, thus creating a greater risk of empyema and pneumonia after chest drain insertion. However, blunt injuries are also reported to be associated with high risk of complications after chest drain insertion. ${ }^{22}$ In view of this, different types of chest injury were not separated. Second, there was a lack of reported risk factors that could potentially trigger pneumonia or empyema in a patient with chest trauma not related to chest drain insertion such as retained pleural fluid or perforated viscus with diaphragmatic tear. Third, antibiotic selection used was different in some studies, particularly in older trials. Often, the choice of antibiotics among studies did not coincide with published guidelines for chest trauma nor directed to commonly cultured pathogens such as gram-positive Staphylococcus aureus. However, most trials used a first generation cephalosporin (cefazolin), which comply with antibacterial guidelines. Hence, to minimize study selection bias, we included all studies comparing prophylactic antibiotic versus placebo despite of their antibiotic rationale.

Finally, our review demonstrates limited recommendations regarding prophylactic antibiotic duration. We found two studies limiting antibiotic administration to 24 hours, ${ }^{12} 16$ with the rest of studies demonstrating patients receiving prophylactic antibiotics until the chest drain was removed. A study by Demetriades et al recommended a single dose prophylactic antibiotic in penetrating chest trauma when compared with a prolonged course. ${ }^{23}$ However, we were unable to find similar studies recommending shorter courses of prophylactic antibiotics. An interesting finding by Maxwell et al showed that resistant organisms were cultured out in chest trauma patients that received prophylactic antibiotics. ${ }^{7}$ Therefore, this finding suggests an increasing pattern of resistant organisms in patient receiving prolonged antibiotics course. Hence, future trails should compare the administration of single dose prophylactic antibiotic versus 24 hours versus prolonged course in the management of chest trauma patient after chest drain insertion.

\section{CONCLUSION}

Our review concludes that administering prophylactic antibiotic for patients with penetrating and blunt chest trauma after chest drain insertion is associated with reduced risk for developing empyema and pneumonia. Further studies should evaluate the optimal type, dose, and duration of antibiotic given to patients with chest trauma.

Contributors FA designed the systematic review, collected data, performed statistical analysis, and has written the article. MQ acted as a second independent data collector. DF acted as a clinical supervisor and helped in evaluating the study.

Funding The authors have not declared a specific grant for this research from any funding agency in the public, commercial or not-for-profit sectors.

Competing interests None declared.

Patient consent for publication Not required.

Provenance and peer review Not commissioned; externally peer reviewed.

Open access This is an open access article distributed in accordance with the Creative Commons Attribution Non Commercial (CC BY-NC 4.0) license, which permits others to distribute, remix, adapt, build upon this work non-commercially, and license their derivative works on different terms, provided the original work is properly cited, appropriate credit is given, any changes made indicated, and the use is non-commercial. See: http://creativecommons.org/licenses/by-nc/4.0/.

\section{REFERENCES}

1. Blyth A. Thoracic trauma. BMJ 2014;348:bmj.g1137.

2. Battle $\mathrm{C}$, Hutchings $\mathrm{H}$, Evans PA. Outcomes in older blunt chest wall trauma patients: a retrospective study. Critical Care 2012;16(Suppl 1):P453-P.

3. Ravi C, McKnight CL, Tube C. Chest tube. StatPearls. treasure Island (fl): StatPearls publishing: StatPearls Publishing LLC, 2018.

4. Ball CG, Lord J, Laupland KB, Gmora S, Mulloy RH, Ng AK, Schieman C, Kirkpatrick AW. Chest tube complications: how well are we training our residents? Can J Surg 2007;50:450-8.

5. LeBlanc KA, Tucker WY. Prophylactic antibiotics and closed tube thoracostomy. Surg Gynecol Obstet 1985;160:259-63.

6. Stone HH, Symbas PN, Hooper CANN. Cefamandole for prophylaxis against infection in closed tube thoracostomy. J Trauma 1981:21:975-7.

7. Maxwell RA, Campbell DJ, Fabian TC, Croce MA, Luchette FA, Kerwin AJ, Davis KA, Nagy K, Tisherman S. Use of presumptive antibiotics following tube thoracostomy for traumatic hemopneumothorax in the prevention of empyema and pneumonia--a multi-center trial. J Trauma 2004:57:742-9.

8. Bosman A, de Jong MB, Debeij J, van den Broek PJ, Schipper IB. Systematic review and meta-analysis of antibiotic prophylaxis to prevent infections from chest drains in blunt and penetrating thoracic injuries. Br J Surg 2012;99:506-13.

9. Moore FO, Duane TM, Hu CKC, Fox AD, McQuay N, Lieber ML, Como JJ, Haut ER, Kerwin AJ, Guillamondegui OD, CK H, Jr. LML, et al. Presumptive antibiotic use in tube thoracostomy for traumatic hemopneumothorax: an eastern association for the surgery of trauma practice management guideline. J Trauma Acute Care Surg 2012;73(5 Suppl 4):S341-4.

10. Moher D, Liberati A, Tetzlaff J, Altman DG, PRISMA Group. Preferred reporting items for systematic reviews and meta-analyses: the PRISMA statement. PLoS Med 2009:6:e1000097.

11. Higgins JPT, Altman DG, Gøtzsche PC, Jüni $P$, Moher D, Oxman AD, Savovic J, Schulz KF, Weeks L, Sterne JAC, et al. The Cochrane collaboration's tool for assessing risk of bias in randomised trials. BMJ 2011;343:d5928.

12. Heydari MB, Hessami MA, Setayeshi K, Sajadifar F. Use of prophylactic antibiotics following tube thoracostomy for blunt chest trauma in the prevention of empyema and pneumonia. J Inj Violence Res 2014;6:91-2. 
13. Grover FL, Richardson JD, Fewel JG, Arom KV, Webb GE, Trinkle JK. Prophylactic antibiotics in the treatment of penetrating chest wounds. A prospective double-blind study. J Thorac Cardiovasc Surg 1977;74:528-36.

14. Mandal AK, Montano J, Thadepalli H. Prophylactic antibiotics and NO antibiotics compared in penetrating chest trauma. J Trauma 1985;25:639-43.

15. LoCurto JJ, Tischler CD, Swan KG, Rocko JM, Blackwood JM, Griffin CC, Lazaro EJ, Reiner DS. Tube thoracostomy and trauma--antibiotics or not? J Trauma 1986:26:1067-72.

16. Cant PJ, Smyth S, Smart DO. Antibiotic prophylaxis is indicated for chest stab wounds requiring closed tube thoracostomy. Br I Surg 1993;80:464-6.

17. Gonzalez RP, Holevar MR. Role of prophylactic antibiotics for tube thoracostomy in chest trauma. Am Surg 1998;64:617-20.

18. Villegas-Carlos F, Vázquez-Martínez AM, Pinedo-Onofre JA, Guevara-Torres L, Belmares-Taboada JA, Sánchez-Aguilar M. [Are antimicrobials useful in closed thoracostomy due to trauma?]. Cir Cir 2009;77:29-32.
19. Nichols RL, Smith JW, Muzik AC, Love EJ, McSwain NE, Timberlake G, Flint LM. Preventive antibiotic usage in traumatic thoracic injuries requiring closed tube thoracostomy. Chest 1994;106:1493-8.

20. Brunner RG, O'NEAL VINSANT G, Alexander RH, Laneve L, FALLON WF. The role of antibiotic therapy in the prevention of empyema in patients with an isolated chest injury (ISS 9-10). J Trauma 1990;30:1148-54.

21. Laws D, Neville E, Duffy J, Pleural Diseases Group, Standards of Care Committee, British Thoracic Society. BTS guidelines for the insertion of a chest drain. Thorax 2003;58 Suppl 2:ii53-9.

22. Walker WE, Kapelanski DP, Weiland AP, Stewart JD, Duke JH. Patterns of infection and mortality in thoracic trauma. Ann Surg 1985;201:752-7.

23. Demetriades D, Breckon V, Breckon C, Kakoyiannis S, Psaras G, Lakhoo M, Charalambides D. Antibiotic prophylaxis in penetrating injuries of the chest. Ann $R$ Coll Surg Engl 1991;73:348-51. 